\title{
Michael, Dowker, Worrell, Banerjee Theorems Extended to Multifunctions
}

\author{
Terrence Anthony Edwards ${ }^{1}$, James Edwards Joseph ${ }^{2}$, Bhamini M. P. Nayar ${ }^{3}$ \\ ${ }^{1}$ Department of Mathematics University of the District of Columbia, Washington DC, USA \\ ${ }^{2}$ Department of Mathematics, Howard University, Washington DC, USA \\ ${ }^{3}$ Department of Mathematics Morgan State University, Baltimore, USA
}

\section{Email address:}

tajedwards@ netscape.net (T. A. Edwards), jjoseph@howard.edu (J. E. Joseph), Bhamini.Nayar@morgan.edu (B. M. P. Nayar)

\section{To cite this article:}

Terrence Anthony Edwards, James Edwards Joseph, Bhamini M. P. Nayar. Michael, Dowker, Worrell, Banerjee Theorems Extended to Multifunctions. American Journal of Applied Mathematics. Vol. 9, No. 2, 2021, pp. 38-43. doi: 10.11648/j.ajam.20210902.11

Received: February 2, 2021; Accepted: February 23, 2021; Published: March 30, 2021

\begin{abstract}
E. Michael, in 1957, proved that the pracompactness is preserved by continuous closed functions from a space onto another. Michael's proof is an immediate consequence of his characterization of paracompact spaces as those spaces with the property that each open cover of the space has a closure preserving refinement. Normality and transfinite induction were used to produce this characterization. J. M. Worrell, in 1985, proved, using the well-ordering principle, that continuous closed images of metacompact spaces are metacompact, as a consequence of a characterization of metacompact spaces he established earlier the same year. C. H. Dowker and R. N. Banerjee have provided the corresponding results for countable paracompactnes and countable metacompactness. In this article we extend these results for continuous, image closed and onto multifunctions. A result due to Joseph and Kwack that all open sets in $Y$ have the form $g(V)-g(X-V)$, where $V$ is open in $X$, if $g: X \rightarrow Y$ is continuous, closed and onto (2006), is extended to image-closed, continuous, multifunctions. Such multifunctions as well as a characterization that a space is paracompact (metacompact) if and only if every ultrafilter of type $P(M)$ converges, proved, in 1918, by Joseph and Nayar, is used to give generalizations of the invariance of paracompactness and metacompactness under continuous closed surjections to multifunctions.
\end{abstract}

Keywords: Multifunctions, Paracpmpact Spaces, Metacompact Spaces

\section{Introduction and Preliminaries}

A multifunction from a set $X$ to a nonemty set $Y$ is a function from $X$ to $2^{Y}-\{\emptyset\}$ where $2^{Y}$ is the power set of $Y$. Denote the collection of multifunctions from $X$ to $Y$ by $\mathcal{M}(X, Y)$. The concept of a multifunction, also known as a set-vlaued function, has been in the lterature for long time and has been used by several researchers to invesitigate different topooical properties. E. Michael proved that continuous closed images of paracompact spaces are paracompact [15]. A space is paracompact if each open cover of the space has a locally finite open refinement. A collection $\mathcal{S}$ of subsets of of a space $X$ is locally finite if for each $x \in X, V \cap S=\emptyset$ for some open set $V$ about $x$ and all but a finite number of $S \in \mathcal{S}$. Michael's proof is an immediate consequence of his characterization of paracompact spaces as those spaces with the property that each open cover of the space has a closure preserving refinement. A family $\mathcal{A}$ of subsets of a space is said to be closure preserving if $c l\left(\bigcup_{B \in \mathcal{M}} B\right)=\bigcup_{B \in \mathcal{M}} c l B$ for every subcollection $\mathcal{M} \subset \mathcal{A}$ [8]. Normality and transfinite induction were used to produce this characterization [15]. A space is metacompact if each open cover of the space has a point finite open refinement. A collection $\mathcal{S}$ of sets of a space $X$ is called point finite if each $x \in X, x \notin S$ for all but finitely many elements $S$ of $\mathcal{S}$. J. M. Worrell proved, using the well-ordering principle, that continuous closed images of metacompact spaces are metacompact.[24]. This was done as a consequence of a characterization of metacompact spaces established in [23]. These theorems, as well as theorems of Dowker and Banerjee are extended to multifunctions, using a different characterization of image-closed multifunction, without using normality, transfinite induction or the wellordering principle [7], [1].

Another generalization of the theorem for the preservation 
of paracompactness and metacompactness by continuous, closed surjections is also provided here using a characterization of paracompact spaces (metacompact spaces) in terms of ultrafilters. It was proved that a space $X$ is paracompact (metacompact) if and only if every ultrafilter of type $P$ (type $M$ ) converges in $X$ [14]. A filter is of type $P(M)$ if every locally ultimately dominating (l.u.d.) (point dominating (p.d.)) subcollection of the filter has non-empty adherence. Definitions of 1.u.d. and p.d. families, ultrafilter of type $P$ (type $M$ ) and related concepts are given in detail later. This result is then extended to multifunctions.

Here, basic definitions and related properties of multifunctions are given. No particular source is cited for these concepts here but are included for completeness and for easy reference for the readers. The image of $A \subseteq X$ (respectively, inverse image of $B \subseteq Y)$ under $\varphi \in \mathcal{M}(X, Y)$ is $\cup_{x \in A} \varphi(x)$ (respectively, $\{x \in X: \varphi(x) \cap B \neq \emptyset\}$ ) and will respectively be denoted by $\varphi(A)$ and $\varphi^{-1}(B)$. If $X$ and $Y$ are topological spaces, and $A \subseteq X, \Sigma A$ will represent the family of open sets which contain $A$. A multifunction $\varphi \in \mathcal{M}(X, Y)$ is upper semi-continuous (u.s.c.) (respectively, lower semi-continuous (l.s.c.)) at $x \in X$ if for each $U \in \Sigma \varphi(x)$ there is a $V \in \Sigma\{x\}$ such that $\varphi(V) \subseteq U$ (respectively, $V \subseteq \varphi^{-1}(U)$ ), where $\Sigma\{x\}$ represents the collection of open sets containing $x$ and $\varphi$ is $u . s . c .($ respectively, l.s.c.) if $\varphi$ is u.s.c. (respectively, l.s.c) at each $x \in X$. It is not difficult to see that $\varphi \in \mathcal{M}(X, Y)$ is u.s.c. (respectively, l.s.c.) if and only if $\varphi^{-1}(B)$ is closed (respectively, open) for each closed (respectively, open) subset $B$ of $Y$. A multifunction $\varphi \in \mathcal{M}(X, Y)$ is continuous if $\varphi$ is both u.s.c and 1.s.c. and $\varphi \in \mathcal{M}(X, Y)$ is said to be image-closed if $\varphi(A)$ is closed for each closed subset $A$ of $X$.

The concept of $\theta$-closure of a set was introduced by Veličko for study of $\mathrm{H}$-closed spaces [21]. If $A \subseteq X$, the $\theta$-closure of $A$, denoted as $c l_{\theta}(A)=\{x \in X: A \cap c l U \neq \emptyset\}$ for each $U \in$ $\Sigma\{x\}$. A set $A$ is $\theta$-closed if $A=c l_{\theta} A$. For a filterbase $\mathcal{F}$, $\theta$-adherence is denoted as $a d h_{\theta} \mathcal{F}$ and $a d h_{\theta} \mathcal{F}=\cap_{\mathcal{F}}\left(c l_{\theta} F\right)$. This concept has been an effective tool for investigators since then [4], [10]. A multifunction $\varphi \in \mathcal{M}(X, Y)$ is strongly subclosed if $\operatorname{adh}_{\theta} \varphi(\Gamma(x)) \subseteq \varphi(x)$ for each $x \in X$ for which $\Gamma(x)=\{V-\{x\}: V \in \Sigma\{x\}\}$ is a filterbase on $X ; \varphi$ is said to be strongly closed if $a d h_{\theta} \varphi(\Sigma\{x\}) \subseteq \varphi(x)$ for each $x \in X$ [10]. It is shown that $\varphi$ is strongly closed if and only if it is strongly subclosed and $\varphi(x)$ is $\theta$-closed for each $x \in X$ [10]. A function $g: X \rightarrow Y$ has strongly subclosed inverse, (respectively, strongly closed inverse, if the relation $g^{-1}: Y \rightarrow X$ is strongly subclosed (respectively, strongly closed). It is not difficult to show that continuous and in fact $\theta$-continuous functions have strongly subclosed inverses [10].

\section{Methods}

The following concepts and methods are used to provide alternate proofs of theorems of Michael and Worrell and then the proofs of theorems of Dowker and Baerjee, extended to multifunctions. One of them is based on the generalization of the characterization of a continuous, closed and onto function provided by Joseph and Kwack to multifunctions [11]. The other method is based on the ultrafilter characterization of paracompactness and metacompactness provided by Joseph and Nayar[14]. These results are extended to multifunctions and details of these characterizations and the extension to multifunctions are given below.

It was shown that a function $g: X \rightarrow Y$ is a closed function if and only if $g(X)$ is closed in $Y$ and $g(V)-g(X-V)$ is open in $g(X)$ for each $V$ open in $X$ [11]. This result is generalized to multifunctions and this generalization is used throughout this article. These results on closed functions and some results based on this characterization, are stated here.

Lemma 2.1. Let $X$ and $Y$ be spaces. Then $g: X \rightarrow Y$ is a closed function if and only if $g(X)$ is closed in $Y$ and $g(V)-g(X-V)$ is open in $g(X)$, whenever $V$ is open in $X$ [11].

Corollary 2.1. Let $X$ and $Y$ be spaces. Then $g: X \rightarrow Y$ is a closed function onto $Y$ if and only if $g(V)-g(X-V)$ is open in $Y$ for each open subset $V$ of $X$ [11].

Corollary 2.2. Let $X$ and $Y$ be spaces and let $g: X \rightarrow Y$ be a continuous, closed function onto $Y$. Then the topology on $Y$ is $\{g(V)-g(X-V): V$ open in $X\}$ [11].

Proof. Note that for each $W$, open in $Y, g^{-1}(W)$ is open in $X$ and $W=g\left(g^{-1}(W)-g\left(X-g^{-1}(W)\right)\right.$. Also for each open subset $V$ of $X, g(V)-g(X-V)$ is open in $Y$.

Next are presented generalizations of the above results to $\mathcal{M}(X, Y)$, proving that $\varphi(V)-\varphi(X-V)$ where $V$ is open in $X$, is open in $Y$, if $\varphi \in \mathcal{M}$ is u.s.c., image-closed and onto.

Theorem 2.1. If $X, Y$ are spaces, then $\varphi \in \mathcal{M}(X, Y)$ is image-closed if and only if 1) $\varphi(X)$ is closed in $Y$ and 2) $\varphi(V)-\varphi(X-V)$ is open in $\varphi(X)$ for each $V$ open in $X$.

Proof. Assume $\varphi$ is image-closed. Clearly $\varphi(X)$ is closed in $Y$. If $V$ is open in $X$ then $\varphi(X)-[\varphi(V)-\varphi(X-V)]=$ $\varphi(V \cup(X-V))-[\varphi(V)-\varphi(X-V)]=\varphi(X-V)$. Hence $\varphi(V)-\varphi(X-V)$ is open in $\varphi(X)$. For the converse, let $F$ be closed in $X, \varphi(X)$ closed in $Y$ and suppose $\varphi(V)-\varphi(X-V)$ is open in $\varphi(X)$ for every open $V$ in $X$. Then $\varphi(X)-\varphi(F)=$ $\varphi(X-F)-\varphi(F)$ and thus $\varphi(F)$ is closed in $\varphi(X)$ and consequently in $Y$.

The proof of the next result follows easily, since for a continuous $\varphi \in \mathcal{M}(X, Y), \varphi^{-1}(V)$ is open in $X$, when $V$ is open in $Y$. Also for a multifunction $\varphi \in \mathcal{M}(X, Y)$, for $W \subseteq Y, W \subseteq \varphi\left(\varphi^{-1} W\right)$. However, we have the following corollary:

Corollary 2.3. If $X, Y$ are spaces, then $\varphi \in \mathcal{M}(X, Y)$ is image-closed, onto and continuous, then $\{\varphi(W)-\varphi(X-W)$ : $W$ open in $X$ \} is open in $Y$.

Theorem 1.1 may be used to give another characterization of u.s.c multifunctions.

Corollary 2.4. If $X, Y$ are spaces, $\varphi \in \mathcal{M}(X, Y)$ is u.s.c. if and only if $\varphi^{-1}(W)-\varphi^{-1}(Y-W)$ is open in $X$ for each $W$ open in $Y$.

Proof. The proof is straightforward, since $\varphi \in \mathcal{M}(X, Y)$ is u.s.c., if and only if $\varphi^{-1}(B)$ is closed for each closed $B \subseteq Y$. Apply the Theorem 1.1 to the inverse multifunction $\varphi^{-1}: Y \rightarrow X$.

A space $X$ is collectionwise normal [8] if for each discrete 
collection $\mathcal{C}$ of non-empty closed subsets of $X$, there is a disjoint collection of open subsets $\mathcal{O}$ of $X$ such that for each $F \in \mathcal{C}$ some $O \in \mathcal{O}$ satisfies $F \subset O$.

A family of subsets of a space $X$ is discrete [8] if for each $x \in X$ has a neighborhood which intersects at most one member of the family. It is to be noted that a discrete family of non-empty closed subsets is a disjoint family of closed sets. Also, if $\left\{A_{\alpha} \mid \alpha \in \Lambda\right\}$ is a collection of pairwise disjoint, closure preserving family of non-empty closed subsets, then it is a discrete collection of non-empty closed sets. For, if for $x \in X$, if $x \in \cup A_{\alpha}$, say, $x \in A_{\beta}$, then $X-\left(\cup_{\alpha \neq \beta} A_{\alpha}\right)$ is an open set containing $x$ and it intersects only $A_{\beta}$. If $x \notin \cup A_{\alpha}$, then $X-\cup A_{\alpha}$ is an open set which does not intersect any $A \alpha$. So, the collectionwise normality is also defined as: A space $X$ is collectionwise normal if for each collection $\mathcal{C}$ of non-empty disjoint, closure preserving closed subsets of $X$, there is a disjoint collection of open subsets $\mathcal{O}$ of $X$ such that for each $F \in \mathcal{C}$ some $O \in \mathcal{O}$ satisfies $F \subset O$. Moreover, if a metacompact space $X$ has the property that for each collection $\mathcal{C}$ of non-empty disjoint closed subsets of $X$, there is a disjoint collection of open subsets $\mathcal{O}$ of $X$ such that for each $F \in \mathcal{C}$ some $O \in \mathcal{O}$ satisfies $F \subset O$, then every discrete family of closed sets also has the property and hence the space is collectionwise normal and paracompact, since a metacompact collectionwise normal space is paracompact. This observation was used in [13]. Relationshsips between collectionwise normality, paracomapctness, metacompactness etc. can be found in many literature on Topology [3], [8].

The characterizations of a closed function, $g: X \rightarrow Y$, stated in Lemma 1.1 and Corollary 1.1 are used to show that a continuous closed onto image of a normal space under the function is normal [11]. The following is true for u.s.c. multifunctions. With the above observations, the following Theorem has the same line of proof as in [13]. It is included here for completeness.

Theorem 2.2. Let $X$ be a collectionwise normal space and $\varphi \in \mathcal{M}(X, Y)$ be u.s.c., image-closed, onto, with the property that $A \cap B=\emptyset$ in $Y$ implies that $\varphi^{-1}(A) \cap \varphi^{-1}(B)=\emptyset$ in $X$. Then $Y$ is collectionwise normal.

Proof. Let $\left\{M_{n}: n \in \Omega\right\}$ be a discrete collection of non-empty closed subsets of $Y$. Then, since $\varphi$ is u.s.c. and onto $Y$, with the property that $A \cap B=\emptyset$ in $Y$ implies that $\varphi^{-1}(A) \cap \varphi^{-1}(B)=\emptyset,\left\{\varphi^{-1}\left(M_{n}\right): n \in \Omega\right\}$ is a family of discrete collection of non-empty closed subsets of $X$. For, for each $y \in Y$, there is an open set $N_{y} \subseteq Y$, with $N_{y} \cap M_{n} \neq \emptyset$ for utmost one $n \in \Omega$. Also for $x \in X$, there is $y \in \varphi(x)$ and for that $y, N_{y} \cap M_{n} \neq \emptyset$ for utmost one $n \in \Omega$. Then $x \in \varphi^{-1}\left(N_{y}\right)$ and $\varphi^{-1}\left(N_{y}\right) \cap \varphi^{-1}\left(M_{n}\right) \neq \emptyset$ for utmost one $n \in \Omega$, with the hypothesis. Since $X$ is collectionwise normal there exist a collection of $\left\{O_{n}: n \in \Omega\right\}$ of disjoint open subsets with $\varphi^{-1}\left(M_{n}\right) \subset O_{n}$ for each $n$. So,

$$
M_{n} \subset \varphi\left(O_{n}\right)-\varphi\left(X-O_{n}\right) .
$$

Moreover,

$$
\begin{aligned}
\left(\varphi\left(O_{n}\right)-\varphi\left(X-O_{n}\right)\right) & \cap\left(\varphi\left(O_{m}\right)-\varphi\left(X-O_{m}\right)\right)=\emptyset \\
n & \neq m .
\end{aligned}
$$

Hence $Y$ is colllectionwise normal.

Corollary 2.5. Let $X$ be a collectionwise normal space and $g: X \rightarrow Y$ be continuous, closed and onto. Then $Y$ is collectionwise normal.

Corollary 2.6. Let $X$ be a normal space and $\varphi \in \mathcal{M}(X, Y)$ be u.s.c., image-closed, onto, with the property that $A \cap B=\emptyset$ in $Y$ implies that $\varphi^{-1}(A) \cap \varphi^{-1}(B)=\emptyset$ in $X$. Then $Y$ is normal.

In the hypotheses of Theorem 1.2 and Corollary 1.6, we assumed that the multifunction $\varphi \in \mathcal{M}(X, Y)$ is u.s.c., image closed, onto and with the property that $A \cap B=\emptyset$ implies that $\varphi^{-1}(A) \cap \varphi^{-1}(B)=\emptyset$ where $A, B \subseteq Y$. In the following theorem we show that such a multifunction is 1.s.c. also.

Theorem 2.3. If $\varphi: X \rightarrow Y$ is an image closed, u.s.c. multifunction onto $Y$, with the property that $A \cap B=\emptyset$ implies that $\varphi^{-1}(A) \cap \varphi^{-1}(B)=\emptyset$ where $A, B \subseteq Y$, then $\varphi$ is 1.s.c. and hence is continuous.

Proof. Let $A \subseteq Y$ be open. Then, in view of Theorem 1.1 $\varphi^{-1}(A)-\varphi^{-1}(Y-A)$ is open in $X$. Note that $(Y-A) \cap A=\emptyset$ and hence, in view of the assumption, $\varphi^{-1}(A)-\varphi^{-1}(Y-$ $A)=\varphi^{-1}(A)$ since $\varphi^{-1}(A) \cap \varphi^{-1}(Y-A)=\emptyset$. Therefore, $\varphi^{-1}(A)$ is open in $X$. Therefore, $\varphi$ is 1.s.c. and since it is also u.s.c., it is continuous. The proof is complete.

In section 2 of this article, some applications of the Lemma 1.1 are given. Several applications of this characterization of closed function exist. However, stated here in this section are just a few: some characterizations of the class of C-compact spaces and the class of functionally compact spaces. A space $X$ is $C$-compact $\mathcal{V}$ such that $\{c l V: V \in \mathcal{V}\}$ covers $A$ [22]. A space $X$ is functionally compact if each open filterbase $\Omega$ on $X$ satisfying $A(\Omega)=I(\Omega)$ is an open set base for $I(\Omega)$ where $A(\Omega)$ is the set of adherent points and $I(\Omega)$ is the set of intersection of all members of $\Omega$ [2].

In section 3, the characterization for image-closed, continuous onto multifunction, developed in section 1 is used to give generalizations of the theorems of Michael, Dowker, Worrell, and Banerjee and prove that if $X$ is paracompact, countably paracompact, metacompact, and countably metacompact and $\varphi \in(X, Y)$ is an image-closed, continuous, multifunction onto $Y$, then $Y$ is, respectively, paracompact, countably paracompact, subsetmetacompact, and countably subsetmetacompact. Then use the same line of proof to deduce that paracompactness, metacompactness, countable paracompatness and countable metacompactness are preserved by continuous, closed and onto functions. Ultrafilter characterizations of these spaces, are used to give other proofs that the above concepts are preserved by continuous, closed onto functions [14]. Also using the fact that every metacompact, collectionwise normal space is praracompact and Theorem 1.2 along with Corollary 3.1, another proof is given to show that paracompactness is preserved by continous, closed and onto functions. independent proofs of these results 
are given in [13].

It is to be noted, as stated in the introduction, that the proofs provided here do not assume normality of the space. Also, using a characterization of normal, countably paracompact spaces by Dowker, it can be shown that the continuous, closed, image of a normal, countably paracompact space is countably paracompact [7], [8].

A cover $\mathcal{D}$ of a space $X$ is a locally finite refinement of a collection $\mathcal{A}$ if each $D \in \mathcal{D}$ is a subset of some member of $\mathcal{A}$, and $\mathcal{D}$ is loally finite.

The applications of Lemma 1.1, indicated in this section, are direct consequences of Lemma 1.1 and results which are already established. They are pointed out here as illustrations of the existence of consequences of Lemma 1.1 producing new properties or characterizations of well-known classes of spaces.

Viglino introduced the class of C-compact spaces, and showed that every continuous function from a C-compact space to a Hausdorff space is closed [22]. This property of Ccompact spaces and the characterization of closed function in Lemma 1.1 give the following property of C-compact spaces.

Theorem 2.4. Let $X$ be a C-compact space and $g$ be a continuous function on $X$. Then (1) $g(X)$ is closed and (2) $g(V)-g(X-V)$ is open in $g(X)$ for every open subset $V$ of $X$.

It is shown that a space $X$ is $C$-compact if and only if all functions on $X$ with strongly subclosed inverses are closed functions [16]. In view of this characterization and Lemma 1.1, a new characterization for C-compact spaces is obtained.

Theorem 2.5. A space $X$ is C-compact if and only if for all functions $g$ on $X$ with strongly subclosed inverses, (1) $\mathrm{g}(\mathrm{X})$ is closed and (2) $g(V)-g(X-V)$ is open in $g(X)$ whenever $V$ is open in $X$.

Dickman and Zame gave the following characterization of a functionally compact space: $A$ space $X$ is functionally compact if and only if every function from $X$ into a Hausdorff space is closed [5]. In view of this, the following new characterization of functionally compact spaces follows as another application of Lemma 1.1.

Theorem 2.6. A space $X$ is functionally compact if and only if for each continuous function on $X,(1) g(X)$ is closed and (2) $g(V)-g(X-V)$ is open in $g(X)$ for every open subset $V$ of $X$.

It is shown that a space $X$ is functionally compact if and only if each function on $X$ with strongly closed inverse is a closed function [12]. Using this result and the Lemma 1.1, the following characterization of functionally compact spaces comes.

Theorem 2.7. A space $X$ is functionally compact if and only if for each function $g$ on $X$ with strongly closed inverse, (1) $g(X)$ is closed and (2) $g(V)-g(X-V)$ is open in $g(X)$ whenever $V$ is an open subset of $X$.

Using the chacaterization of image-closed multifunction given in the Theorem 1.1 above and considering that for each function $g: X \rightarrow Y, g^{-1}: Y \rightarrow X$ is a multifunction, the characterizations for C-compact spaces and functionally compact spaces given in Theorems 2.2 and 2.4 can be extended to multifunctions as follows:

Theorem 2.8. A space $X$ is C-compact if and only if for all multifunctions $\varphi \in \mathcal{M}(X, Y)$ with strongly subclosed graphs, (1) $\varphi(X)$ is closed and (2) $\varphi(V)-\varphi(X-V)$ is open in $\varphi(X)$ whenever $V$ is open in $X$.

Theorem 2.9. A space $X$ is functionally compact if and only if for all multifunctions $\varphi \in \mathcal{M}(X, Y)$ with strongly closed graphs, (1) $\varphi(X)$ is closed and (2) $\varphi(V)-\varphi(X-V)$ is open in $\varphi(X)$ whenever $V$ is open in $X$.

\section{Results}

Here, are presented proofs of extensions of Theorems of Michael, Dowker, Worrell, and Bnerjee for $\mathcal{M}(X, Y)$. Corresponding to each open cover of $Y$, there is an open cover $\theta$ of $X$ and sets of the form $\varphi(V)-\varphi(X-V)$ where $V \in \theta$, is a cover of $Y$ if $\varphi \in \mathcal{M}(X, Y)$ is continuous image-closed and onto $Y$. We introduce the following definition to fecilitate the multifunction generalaization of continuous closed invariance of metacompactness.

Definition 3.1. A space $X$ is called compact-metacompact if for each open cover $\mathcal{S}$ of $X$, there is an open refinement $\mathcal{R}$ of $\mathcal{S}$ and a collection of compact subsets $\mathcal{T}$ of $X$ such that $R \cap T=\emptyset$ for all but finitely many $R \in \mathcal{R}, T \in \mathcal{T}$.

Note that every metacompact space is compactmetacompact since the collection $\{\{x\}: x \in X\}$ is a collection of subsets which will satisfy the condition of compact-metacompactness, if the space is metacompact. Considering the image of a metacompact space under multifunctions, for each $x \in X, \varphi(x)$ is a set, not necessarily a single point set. Moreover, for each $x \in X, \varphi(x)$ is compact since $\varphi$ is continuous, image closed and onto. Thus, here we prove that the image of a metcompact space under a multifunction is compact-metacompact, first and then deduce that the image of a metacompact space under a continuous, image closed onto function is metacompact.

The next theorem consists of extensions of results of Michael (Worrell) to multifunctions.

Definition 3.2. Let $X, Y$ be spaces with $X$ paracompact (metacompact) and let $\varphi \in \mathcal{M}(X, Y)$ be continuous image-closed and onto. Then $Y$ is paracompact (compactmetacompact).

Proof. With the assumptions on the $\varphi \in \mathcal{M}(X, Y)$, each open cover of $Y$ gives an open cover of $X$ and let $\theta$ be such an open cover of $X$. So, $\theta$ has a locally finite (point finite) refinement. Let $\mathcal{B}$ be a locally finite (point finite) refinement of $\theta$. Then for each $x \in X$, there is a $V \in \Sigma\{x\}$ such that $V \cap B=\emptyset(x \notin B)$ for all but finitely many $B \in \mathcal{B}$ Therefore, for all but finitely many $B \in \mathcal{B}, V \subseteq X-B(x \in$ $X-B)$ and hence $\varphi(V) \subseteq \varphi(X-B)(\varphi(x) \subseteq \varphi(X-B))$. Hence $(\varphi(V)-\varphi(X-V)) \cap(\varphi(B)-\varphi(X-B))=\emptyset$ $(\varphi(x) \cap(\varphi(B)-\varphi(X-B))=\emptyset$ for all but finitely many $B \in \mathcal{B}$. Since $\varphi$ is an image closed multifunction onto $Y$, $\{\varphi(B)-\varphi(X-B)\}$ is a locally finite refinement of the open cover of $Y$ associated with the cover $\theta$ of $X$ and also it satisfies the condition of compact- metacompactness of $Y$. Hence $Y$ 
is paracompact (compact-metacompact.) Thus the proof is complete.

Corollary 3.1. Let $X, Y$ be spaces with $X$ paracompact (metacompact) and let $\varphi \in \mathcal{M}(X, Y)$ be continuous imageclosed onto function. Then $Y$ is paracompact (metacompact).

Proof. The proof for paracompactness is the same as above. For metacompactness, $\varphi$ is a function and hence $\varphi(x)$ is a single point and for each $y \in Y$ there is an $x \in X$ such that $\varphi(x)=y$. The proof follows.

Replacing "open cover" with "countable open cover", the same method as above will give theorems of Dowker and Banerjee [7], [1].

Theorem 3.1. Let $X, Y$ be spaces with $X$ countably paracompact (countably metacompact) and let $\varphi \in \mathcal{M}(X, Y)$ be continuous image-closed onto multifunction. Then $Y$ is countably paracompact (subsetmetacompact).

Corollary 3.2. [Michael (Dowker)((Worrell)) (((Banerjee)))]. If $g: X \rightarrow Y$ is a continuous closed onto function and $X$ is paracompact (countably paracompact) ((metacompact)) (((countably metacompact))), then $Y$ is paracompact (countably paracompact) ((metacompact)) (((countably metacompact))).

Remark 3.1. We use Theorem 1.2, Theorem 3.1, Corollary 3.1 and the fact that a space is paracompact if and only if it is metacompact and collectionwise normal to prove the following [8]. Thus another proof is obtained for the result that the image of a paracompact space under continuous, closed and onto function is paracompact.

The following theorem is proved in [14]. There, characterizations of paracompactness, metacompactness, paralindel' of spaces and meta-Lindel'of spaces are given in terms of ultrafilters. Proofs, in cases of pracompact spaces and metacompact spaces are given here for completeness. The following definitions and results are used in the proofs and can be found in [14].

A filterbase $\Omega$ is defined to be of type $P$ if each locally ultimately dominating (l.u.d.) filter subbase coarser than $\Omega$ has non-empty adherence. A family is locally ultimately dominating (l.u.d) if for each $x \in X$ there is an open set about $x$ contained in all but finitely many elements of $\Omega$. A space $X$ is metacompact if and only if every filter (or closed filter) of type $M$ on $X$ has non-empty adherence. A filter on a space is of type $M$ if every point dominating (p.d.) subcollection of the filter has nonempty adherence. A collection $\Omega$ of subsets of a space is point dominating (p.d.) if each $x \in X$ is a member of all but finitely many members of $\Omega$. An ultrafilter $\mathcal{U}$ is said to be a of type $P(M)$ if it contains a filter of type $\mathrm{P}(\mathrm{M})$. In Theorem 1 of the referenced paper, it is proved that If $\mathcal{U}$ is a filter which contains a filter of type $P(M)$, then $\mathcal{U}$ itself is a filter of of type $P(M)$ [14]. For completeness, the result is included here as a lemma.

Lemma 3.1. If $\mathcal{U}$ is a filter which contains a filter of type $P$ $(M)$, then $\mathcal{U}$ itself is a filter of of type $P(M)$ [14].

Proof. Suppose $\mathcal{U}$ is a filter which contains a filter $\mathcal{B}$ of type $P(M)$. Consider a l.u.d. (p.d.) subcollection $\mathcal{C}$ of $\mathcal{U}$. Then $\mathcal{C} \cap \mathcal{B}$ is a l.u.d. (p.d.) subcollection of $\mathcal{B}$ and hence has nonempty adherence. Therefore, $\mathcal{C}$ has a non-empty adherence and hence $\mathcal{U}$ is a filter of type $P(M)$.

Theorem 3.2. The following are equivalent:

1. $X$ is paracompact (metacompact);

2. Every ultrafilter of type $\mathrm{P}(\mathrm{M})$ converges [14].

Proof. (1) $\Longrightarrow$ (2). Clear from the fact that every ultrafilter of type $\mathrm{P}(\mathrm{M})$ has nonempty adherence and every ultrafilter converges to each point of its adherence.

$(2) \Longrightarrow(1)$. Let $\mathcal{U}$ be a filter of type $P(M)$ on $\mathrm{X}$ and $\mathcal{W}$ be an ultrafilter containing $\mathcal{U}$. Then $\mathcal{W}$ is an ultrafilter of type $P(M)$ and hence converges in $X$, inview of the assumption. Hence the filter $\mathcal{U}$ has non-empty adherence and so, $X$ is paracompact (metacompact).

In the following, the previous theorems are extended to multifunctions using ultrafilters and then deduced for single valued functions. The following Lemma is given.

Lemma 3.2. If an ultrafilter on $X$ converges to a compact set, then it converges to a single point.

Proof. Suppose $A$ is a compact subset of $X$ such that an ultrafilter $\mathcal{U} \rightarrow A$. If for each $x \in A$, there is a $V_{x} \in \Sigma\{x\}$ and $U \in \mathcal{U}_{x}$ such that $U_{x} \cap V_{x}=\emptyset$. Then there is a finite set $B \subset A$ such that $A \subset \cup_{x \in B} V_{x}=V$ and $U=\cap_{x \in B} U_{x}$ with $U \in \mathcal{U}$. That is, there is a $V \in \Sigma A$ and $\mathrm{U} \in \mathcal{U}$ such that $V \cap U=\emptyset$, which is a contradiction since $\mathcal{U} \rightarrow A$. Thus there is an $x \in A$ such that $\mathcal{U} \rightarrow x$.

Theorem 3.3. Let $X, Y$ be spaces with $X$ paracompact (metacompact) and let $\varphi \in \mathcal{M}(X, Y)$ be continuous, imageclosed and onto. Then every ultrafilter of type $P(M)$ on $Y$ converges.

Proof. Let $\mathcal{U}$ be an ultrafilter of type $P(M)$ on $Y$. Then $\varphi^{-1} \mathcal{U}$ is an ultrafilter on $X$. Let $\mathcal{V}$ be a l.u.d. (p.d.) subcollection coarser than $\varphi^{-1} \mathcal{U}$. Since $\varphi$ is image-closed and onto, it follows that $\varphi(\mathcal{V})$ is a l.u.d. (p.d.) collection coarser than $\mathcal{U}$. Hence, $\operatorname{adh} \varphi(\mathcal{V}) \neq \emptyset$; so $\operatorname{adh} \mathcal{V} \neq \emptyset$. Therefore, $\varphi^{-1} \mathcal{U}$ is an ultraifilter of type $P(M)$, and $\operatorname{adh} \varphi^{-1} \mathcal{U} \neq \emptyset$. Since $\varphi$ being continious, image-closed and onto, and $\varphi(x)$ is a compact subset of $Y, \mathcal{U} \rightarrow y \in \varphi(x)$, in view of Lemma 3.4.

Corollary 3.3. Let $X, Y$ be spaces with $X$ paracompact (metacompact) and let $g: X \rightarrow Y$ be continuous, closed and onto. Then $Y$ is paracompact (metacompact).

\section{Discussion and Conclusion}

In this article shorter proofs of theorems of Michael and Worrell are given, It is obtained by extending the characterization of continuous, closed and onto function provided by Joseph and Kwack to multifunctions [11]. Filter characterizations of paracompactness and metacompactness paved the way for ultrafilter characterizations of these classes of spaces [14]. These characterizations provided considerably shorter and straight forward proofs of theorems of Micahel and Worrell.

Paracompactness, metacompactness, collectionwise normality, discrete collection of sets, a family being locally finite or point finite etc. are classic topological concepts and have been topics and tools of investigation for decades. There exist numerous literature on these topics. Following are just a 
few of them $[6,8,9,17-20]$. The definitions and basic results of concepts which are generally available in literature are not given with citation here, however, the definitions and the basic results are stated here for completeness and easy access for the readers. The authors of this article do not claim authorship of them. When a definition of a new concept is given, it is explicitely stated. Whenever a result is stated from another source, care is taken to give appropriate citation of each of them.

\section{References}

[1] R. N. Banerjee, Closed maps and countable metacompact spaces, J. London Math. Soc. 2 No. 8 (1974), 49-50.

[2] A. Bella, A note on functionally compact spaces, Proc. Amer. Math. Soc. 98 No. 1 (1986), 507 - 512.

[3] S. W. Davis, Topology, McGraw Hill Companies, The Walter Rudin Student Series in Advanced Mathematics (2005)

[4] R. Dickman and J. R. porter, $\theta$-closed subsets of Hausdorff spaces, Pacific Journal of Mathematics, 59 No. 2 (1975), 407-415.

[5] R. F. Dickman and A . Zame, functionally compact spaces, Pacific J. Math. 31 (1969), 303-311.

[6] J. Dieudonné, Une généralisation des espaces compacts, J. Pures Appl. 23 (1944), 65-76.

[7] C. H. Dowker, On countably paracompact spaces, Canad. J. Math. 3 No. 2 (1951), 219 - 224.

[8] R. Engelking, General Topology, PWN-Polish Scientific Publishers, Warszawa, Poland, (1977).

[9] T.R. Hamlett, D. Rose and D. Jankovic Paracompactness with respect to an ideal, International Journal of Mathematics and Mathematical Sciences 20 No. 3 (1997), 433-442.

[10] J. E. Joseph, Multifunctions and graphs, Pac. J. Math. 79 (1978), 509-529.
[11] J. E. Joseph and M. H. Kwack, A note on closed functions, Missouri J. Math. Sci. 18 (1), 59 -61, Winter 2006.

[12] J. E. Joseph, M. H. Kwack and B. M. P. Nayar, Sequentially functionally compact and sequentially $C$ compact spaces, Scientae Mathematicae 2 (2) (1999), 187-194.

[13] J. E. Joseph and B. M. P. Nayar, New Proofs of Theorems of Michael and Worrell, Journal of Advanced Studies in Topology Vol 8: 1 (2017), 21 - 23.

[14] J. E. Joseph and B. M. P. Nayar, Ultrafilter chracerizations of paracompact, metacompact, paralindel'of and metaLindel'of spaces , International Journal of Pure and Applied Mathematics Vol. 118, No. 4 (2018) 1001-1005.

[15] E. Michael, Another note on paracompactness, Proc. Amer. Math. Soc. 8 (1957) 822-828.

[16] B. M. P. Nayar, A remark on C-compact spaces, J. Austral. Math. Soc. (Series A) 64 (1998), 327 - 328.

[17] K. Sakai, Geometric Aspects of General Topology Springer Nature (2013).

[18] T. B. Singh, Introduction to Topology, Springer Science and Business Media LLC (2019).

[19] A. H. Stone, Paracompactness and product spaces, Bull. Amer. Math.Soc. 54 (1948), 977-992.

[20] J.E. Vaughan, Linearly ordered collection of paracompactness, Proceedings of American Math. Soc. 24 (1970), 186-192.

[21] N. V. Veličko, H-closed topological spaces, Math.Sb (N. S) 70(112) (1966), 98 - 112 (Russian), ( Amer. Math Soc. Transl. 78 (2) (1968), 103-118).

[22] G. Viglino, C-compact spaces, Duke Math. J. 36 (1969), 761-764.

[23] J. M. Worrell, A characterization of metacompact spaces, Port. Math. 85 Fas. 3 (1985), 172-174.

[24] J. M. Worrell, The closed continuous images of metacompact spaces, Port. Math., 85 Fas. 3 (1985), 175179. 